\title{
Syllables are Retrieved before Segments in Mandarin Chinese Spoken Production: An ERP study
}

\author{
Chen Feng ${ }^{2}$, Yuan Yue ${ }^{2}$, Qingfang Zhang ${ }^{1 *}$ \\ ${ }^{1}$ Department of Psychology, Renmin University of China, Beijing, 100872, China \\ ${ }^{2}$ Key Laboratory of Behavioral Science, Institute of Psychology, Chinese Academy of Sciences, Beijing, 100101, China \\ qingfang.zhang@ruc.edu.cn
}

\begin{abstract}
Languages may differ regarding the functional units of word-from encoding in spoken word production. It is widely accepted that segments are the primary units in Indo-European languages. However, it is controversial what are the functional units (syllables or segments) in Chinese spoken word production. In the present study, Mandarin Chinese speakers named pictures while ignoring distractor words presented simultaneously, which were share atonal syllables, initial segments or final segments with the name of the target pictures or were unrelated. Behavioral results showed that naming latencies in the three phonologically related conditions were significantly shortened in comparison with unrelated condition. EEG data indicated that syllable-related condition modulated ERPs in the time window of $320 \mathrm{~ms}$ to $500 \mathrm{~ms}$, initial segment-related condition modulated ERPs from $370 \mathrm{~ms}$ to $420 \mathrm{~ms}$, while final segment-related condition modulated ERPs from $400 \mathrm{~ms}$ to $450 \mathrm{~ms}$. Onset latencies for syllable, initial segments, and final segments priming effects were $322 \mathrm{~ms}, 368 \mathrm{~ms}$, and $408 \mathrm{~ms}$, respectively. Our findings firstly provide a relative temporal course of syllable and segments encoding in Chinese spoken naming: Syllables are retrieved $46 \mathrm{~ms}$ before segments, and they constitute the primary processing units at the early stage of word-form encoding. Furthermore, segments and their order are retrieved incrementally from left to right in producing Chinese spoken words.
\end{abstract}




\section{Introduction}

Speech production involves conceptual preparation, lexical selection, word-form encoding, and articulation processes. In the model of language production proposed by Levelt and colleagues (Levelt, Roelofs \& Meyer, 1999), word-form encoding consists of segmental and metrical retrievals, and then both of them combined into a syllable by syllabification process. The role of sub-lexical units in this process such as the phoneme, syllable or mora has received much attention (Chen, Lin \& Ferrand, 2003; O’Seaghdha, Chen \& Chen, 2010; Schiller, 1998, 1999, 2000; Verdonschot et al., 2011).

Evidence for phonemes as primary units in spoken production comes from various Indo-European languages, such as Dutch (Meyer, 1991; Meyer, Schriefers, 1991; Roelofs, 1999), English (Schiller, 2000; Damian, Dumay, 2007; Collins, Ellis, 1992; Damian, Dumay, 2009), Russian (Timmer, Ganushchak, Mitlina, \& Schiller, 2014; Jouravlev, Lupker, \& Jared, 2014) and Persian (Timmer, Vahidgharavi, \& Schiller, 2012), with different tasks including implicit priming paradigm, masked priming paradigm or phonemes repetition task. For example, in a masked form priming paradigm, Dutch participants named bisyllabic words which were preceded by visual masked primes. When primes shared the initial segment(s) with the target, naming latencies were significantly shorter than the segments not shared condition. Damian and Dumay (Damian, Dumay, 2007) reported that when participants produced adjective-noun phrases in response to colored objects, a single-phoneme overlap between the color and object name facilitated responses (e.g., red rope), even when the overlapping phoneme occupied different positions within the words (Damian, Dumay, 2009). These findings support that phonemes are the primary functional units of word-form encoding.

The claim that phonemes are primary phonological encoding units in Indo-European languages is plausible because the orthographies of these alphabetic languages explicitly code for phonemes via letter or letter combinations. What about non-alphabetic languages such as Chinese? Chinese has a logographic writing script in which orthographic representations code not for phonemes, but instead characters map onto syllables. Mandarin Chinese has approximately 400 atonal syllables, and all these syllables have a very straightforward structure, and no resyllabification is required in speaking. This variation in phonological properties suggests that it cannot be directly assumed that phonemes are equally important across all languages and therefore, it raises the necessity to investigate the specific encoding mechanisms of different languages.

Recently emerging evidences offer some support for the possibility that for Chinese speakers, syllables rather than phonemes constitute primary units of word-form encoding. This was supported by earlier behavioural findings of a reliable syllabic priming effect but the null effect of phoneme priming during word production of Mandarin (Chen, Lin \& Ferrand, 2003; O’Seaghdha, Chen \& Chen, 2010; Chen, Chen \& Dell, 2002; Zhang, 2008; You, Zhang, Verdonschot, 2012; Wang, Wong, Wang \& Chen, 2017; Chen, O’Seaghdha \& Chen, 2016; Chen, Chen, 2013). The role of shortly presented prime words which matched or mismatched their first syllable structure with the spoken Chinese words was examined in a masked priming task (Chen, Lin \& Ferrand, 2003). They found that CV (C represents consonant, and V represents vowel) targets were named faster when preceded by CV primes compared to CVG primes (G represents glide sound), while the opposite pattern was obtained for CVG targets. Similar results were reported in both word reading and picture naming (You, Zhang, Verdonschot, 2012). Most recently, the syllabic priming effect was replicated but little evidence of phonemic priming (Chen, O’Seaghdha \& Chen, 2016); in fact, phonemically related primes elicited a slightly inhibitive effect when compared to phonemically unrelated primes. In a delayed picture naming study with electrophysiological measures (Wang, Wong, Wang \& Chen, 2017), participants were required to name pictures upon a response cue. Each two consecutively presented pictures formed a pair of prime and target, which shared the same 
word-initial syllable or phoneme, or were unrelated. Syllable shared elicited an effect in the time windows of 200-400 ms and 400-600 ms, where no significant ERP effects were found for phoneme shared.

However, a few recent studies using other tasks reported sub-syllabic priming effects in Chinese spoken word production. Using a picture-word interference task, it was found significant facilitation effects when the target and the distractor word shared the syllable, or when only the final segments was shared (Wong, Chen, 2008), reflecting that subsyllabic units might be a possible candidate of functional unit. Furthermore, studies electrophysiological measures found difference between phonemes shared and not shared condition(Qu, Damian \& Kazanina, 2012; Yu, Mo, \&Mo, 2014). Using a phoneme repetition task, $\mathrm{Qu}$ and colleagues reported that phoneme repetition modulated ERP at $200 \mathrm{~ms}$ after pictures onset when color and object name shared the initial phoneme. A similar finding was observed from an implicit priming paradigm in which showed that phoneme repetition evoked more positive ERPs in the time window of $180 \mathrm{~ms}$ to $300 \mathrm{~ms}$ post target pictures (Yu, Mo, \&Mo, 2014). Also, in a fMRI study (Yu, Mo, Li \& Mo, 2015), distinctive brain regions have been found to exhibited neural adaptation effect for phoneme (bilateral basal ganglia) and syllable (bilateral superior temporal gyrus). Taken together, researchers suggest that phoneme constitute fundamental units of phonological encoding even in Chinese spoken word production.

These findings present a complex picture, but a plausible scenario is that speakers of all language mentally represent phonemes and syllables, but attribute differential importance and temporal courses depending on the target language. To explain these various findings, O'Seaghdha et al. proposed a proximate unit principle (O'Seaghdha, Chen \& Chen, 2010; O’Seaghdha, 2015) in which assumes that phonological units below the word vary across languages. This principle has been integrated into the influential spoken production model, i.e., WEAVER++, which assumes that in its original form that phonological encoding in languages involves a parallel activation of phonemic segments and metrical frames in spoken production system, followed by prosodification, which concerns serial content-to-frame association and syllabification (Roleofs, 2015). For Chinese speakers, atonal syllables are associated to the tonal frame serially as the first selectable units below the lexeme (O'Seaghdha, Chen \& Chen, 2010; Roleofs, 2015). After the retrieval of syllables, the phonemes within a syllable are retrieved in parallel and linked to their position in the syllabic frame. This principle has also been validated through a computer simulation (Roleofs, 2015).

A critical factor to distinguish these versions is the time courses of different phonological units. For Chinese spoken production, the retrieval of syllables would precede phonemes, and phonemes within a syllable are processed simultaneously according to a parallel mechanism (Roleofs, 2015). Addressing these questions requires a high time resolution technique such as EEG. Although there have been evidences from ERP studies showing involvement of segment in a quite early stage, i.e., around $200 \mathrm{~ms}$ (Qu, Damian \& Kazanina, 2012; Yu, Mo, \&Mo, 2014), it is possible to reflect a processing on syllable as shared segments also increases the similarity between two syllables (Wang, Wong, Wang, Chen, 2017). Previous EEG study in Chinese examined the time course of syllables retrieval or phonemes retrieval separately (Wang, Wong, Wang, Chen, 2017; Qu, Damian \& Kazanina, 2012; Yu, Mo, \&Mo, 2014). It is risky to infer the interval between syllabic and phonemic encoding in spoken production via comparisons among studies due to different tasks and different participants involved in studies (Laganaro, M., Valente, A., \& Perret, 2012; Roelofs, A., \& Shitova, 2017). As a consequence, it is critical to include syllables shared condition as well as phonemes/segments shared condition in an experiment for identical participants. In a delayed primed picture naming study reported by Wang and colleagues (Wang, Wong, Wang \& Chen, 2017), each two consecutive pictures implicitly formed a pair of prime and target, whose names shared the same word-initial atonal syllable or the same word-initial segments, or were unrelated in the control conditions. Participants were asked to first prepare and later produce disyllabic Chinese words upon picture prompts when a response cue presented. They found a widely distributed positivity in the 200- to 400-ms 
interval and an anterior positivity in the 400- to 600-ms interval for syllables shared condition, while the null effect for word-initial segments shared condition. There are two possibilities to interpret the null finding of word-initial segments effect. First, there was only one single phoneme shared between primes and targets in the word-initial segments shared condition. The activation of single phonemes was too weak to be detectable. Second, the pictures as primes in combination with a delayed naming task might be insensitive to phonemic activation of distractor pictures. However, using a typical picture-word interference paradigm (PWI), an effect of sharing word-initial segments on naming latencies was observed consistently in several studies (Chen, Chen, 2013; Wang, Wong \& Chen, 2018; Yue, Zhang, 2015). By contrast, phonemes priming effect was absent in other tasks such as phoneme repetition task and masked priming task (Wang, Wong, Wang, Chen, 2017; Chen, O’Seaghdha \& Chen, 2016; Chen, Chen, 2013, Qu, Damian \& Kazanina, 2012). We thus assume that the PWI paradigm is more sensitive to phonemic activation in spoken word production than other tasks.

In this article, we address the limitations of previous studies. We used a typical picture-word interference task, and we included an atonal syllable-related condition, an initial segment-related condition sharing two first phonemes with targets, a final segment-related condition sharing two final phonemes with targets, and unrelated condition between distractors and targets. We recorded both the behavioural and electroencephalography (EEG) signals concurrently. Data from each distractor type would be compared with the unrelated condition. According to the proximate unit principle, we expect an early syllable priming effect and a later initial segment or final segment priming effect, and a parallel time course of initial and final segment processing.

\section{Methods}

\section{Participants}

Twenty undergraduate and graduate students (4 males, average 22.05 years; range 18- 28 years; all right handed) participated in the experiment. They were recruited from Beijing Forestry University, China Agriculture University, and Beijing Science and Technology University. All were native Mandarin Chinese speakers with normal or corrected- tonormal vision. They were paid for 80 yuan (i.e., around 12 USD) for participation. Informed consent was obtained from each participant before the experiment. This study followed the ethical procedures for the protection of human participants in research and was approved by the ethics committee of the Institute of Psychology, Chinese Academy of Science.

\section{Materials}

Eighteen black-on-white line drawings of common objects with monosyllabic names were chosen as targets which were selected from Zhang and Yang's picture naming database in Chinese ${ }^{49}$. All of target names had a CVN syllabic structure (C represents consonant, V represents vowel, and $\mathrm{N}$ represents nasal), e.g., 镜 (/jing4/). Each target was paired with four single character distractors. whose names shared an atonal syllable (same segmental composition but different tone, e.g., 景, /jing3/; hereafter named as syllabic-related condition), an initial segment (same consonant and vowel but different tone, e.g., 姐, /jie3/; hereafter named as initial segment-related condition), a final segment (same vowel and nasal but different tone, e.g., 倾, /qing1/; hereafter named final segment-related condition), and no any phonological overlap (e.g., 捞, /lao1/; hereafter named unrelated condition) with target. (see Fig 1.A). Semantic or orthographic overlap between distractors and targets was avoided. Frequencies of monosyllabic words (Modern Chinese Frequency Dictionary, 1986) and stroke numbers were matched across conditions (Frequency: $F(3,68)=0.001, p=1$; Stroke number: $F(3,68)=0.1, p=0.96)$. All stimuli are presented in Supplementary Material (as a supplementary file). 
In order to counterbalance the trials of phonologically related and unrelated condition, a set of 9 pictures was used as fillers and paired with four unrelated single characters. Consequently, half distractor-target pairs were phonologically related, and half were phonologically unrelated. In addition, there were 6 practice trials in which 3 pictures was paired with one unrelated single character, and each pair was presented twice.

\section{Design}

The experiment adopted a factor of distractor type (syllable-related, initial segment-related, final segment-related, and unrelated) as a within-participants design. Because of limitations on available items, we manipulated the repetition exactly in the same way in J.-Y. Chen et al. (Chen, Lin, \& Ferrand, 2003) and Wang et al. (Wang, Wong, Wang \& Chen, 2017) Each block includes 6 practice trials, 72 target trials (18 targets paired with four different kinds of distractors), and 36 filler trials (9 fillers paired with four unrelated distractors), resulting in 114 trials. Each repetition was set in one block, and two repetitions result in two blocks and 228 trials in total. The order of pictures (targets and fillers) within a block was pseudo-randomized to prevent targets from repeating within five trials. A new sequence was generated for each participant and each block.

\section{Apparatus}

The experiment was performed using E-Prime Professional Software (Version 1.1; Psychology Software Tools). Participants were seated in a quiet room approximately $70 \mathrm{~cm}$ from a 21 inch CRT computer screen with a refresh rate of $100 \mathrm{~Hz}$. Naming latencies were measured from target onset using a voice-key, connected to the computer via a PST Serial Response Box.

\section{Procedure}

Participants were tested individually in front of a computer in a sound-proof room. Participants firstly were asked to familiarize themselves with the 30 pictures by viewing each for $2000 \mathrm{~ms}$ with the picture name printed below each picture. After the learning phase, participants received a picture naming test without names presented. When all pictures were named correctly, the experimental blocks were administered, comprising 114 trials in each block.

Each trial involved the following sequence: a fixation '+' presented at the center of the screen for 500ms, followed by a blank screen lasted for $300 \mathrm{~ms}$, then a target picture superposed with a distractor were presented. Participants were instructed to ignore the distractors and name the pictures as accurately and fast as possible. An inter-trial interval of $1000 \mathrm{~ms}$ concluded each trial. Naming latencies were measured from the onset of the picture. The experiment took about 25 minutes in total, and there was a short break between two blocks.

\section{EEG recordings and analyzing}

The EEG was recorded via a Neuroscan amplifier (Neuroscan SynAmps) through 66 electrodes located at the standard 10-20 scalp sites secured in an elastic cap (Electro Cap International). The vertical electro-oculogram (VEOG) was monitored with electrodes fixed above and below the left eye. The horizontal EOG (HEOG) was recorded by a bipolar montage using two electrodes placed on the right and left external cantus. The left mastoid electrode served as reference. Impendences of all electrodes were kept below $5 \mathrm{k} \Omega$. Electrophysiological signals were amplified with a band-pass filter of 0.05 and $70 \mathrm{~Hz}$ and digitized continuously at a rate of $500 \mathrm{~Hz}$.

The Neuroscan 4.3 software was used to analyze the ERP data. Waveforms of all single-trials were visually inspected firstly and epochs contaminated by eye movements, electrode drifting, amplifier blocking and EMG artefacts or other noise were rejected. The EEG data were re-referenced off-line to the average of both mastoids and filtered off-ling 
using a $0.1 \mathrm{~Hz}$ high-pass filter and $30 \mathrm{~Hz}$ low-pass filter. The EEG data were segmented from $100 \mathrm{~ms}$ before to $500 \mathrm{~ms}$ after the onset of the pictures, with baseline correction ranging from -100 to $0 \mathrm{~ms}$ preceding picture onset. Epochs with amplitudes exceeding $\pm 100 \mu \mathrm{v}$ were rejected. The remained epochs were considered in ERP analyses. For each distractor type, mean amplitudes of the ERP waveforms were analyzed. Nine regions of interest (ROIs) were selected, i.e., left-anterior (F3, F5, FC3), mid-anterior (Fz), right-anterior (F4, F6, FC4), left-central (C3, C5, CP3), mid-central $(\mathrm{Cz})$, right-central $(\mathrm{C} 4, \mathrm{C} 6, \mathrm{CP} 4)$, left-posterior (P3, P5, PO3), mid-posterior (Pz), and right-posterior (P4, P6, PO4).

\section{Results}

\section{Behavioural Results}

Incorrectly named trials (1.5\%), latencies shorter than $500 \mathrm{~ms}$ or longer than $1500 \mathrm{~ms}(2.5 \%)$ or beyond 3 SDs (1.6\%) were excluded from the analysis. The mean naming latencies for the syllable-related condition were $705.1 \mathrm{~ms}(\mathrm{SE}=3.8$ $\mathrm{ms}), 730 \mathrm{~ms}(\mathrm{SE}=4.2 \mathrm{~ms})$ for the initial segment-related condition, $731.8 \mathrm{~ms}(\mathrm{SE}=4.27 \mathrm{~ms})$ for the final segmentrelated condition, and $745.6 \mathrm{~ms}(\mathrm{SE}=4.26 \mathrm{~ms})$ for the unrelated condition.

The data were analyzed using a linear mixed effects model (Bates, 2005; Baayen, Davidson \& Bates, 2008) that included fixed effects of distractor type (syllable-related, initial segment-related, final segment-related, and unrelated) and block, by-participant and by-item random intercepts and random slope. According to the guideline proposed by Barr and colleagues (Barr, Levy, Scheepers \& Tilfor, 2013), the maximal random effects structure was constructed to obtain a more reliable estimation. For example, the model of the omnibus estimation on latency was:

\section{Latency $\sim($ distractor type + block $+(1+$ distractor type + block $\mid$ Subject $)+(1+$ distractor type + block $\mid$ Item $)$}

The lmer () function of the lme4 package was used to estimate fixed effects and parameter estimation of the LMM. The degree of freedom and $p$-values were computed using ANOVA () function of the lmer test package with Satterthwaite approximations. These analyses were conducted using the free software R (R Development Core Team, 2009)

For naming latencies, the main effect of distractor type was significant, $F_{(3,21.4)}=16.98, p<0.001$, as well as the main effect of block $\left(F_{(3,19.4)}=12.43, p<0.001\right)$, whereas the interaction between distractor type and block was not significant $\left(F_{(6,3942.8)}=1.58, p=0.15\right)$, Multiple comparisons ( $p$ values with $f d r$ correction) was then conducted in each phonologically related condition. All the three phonologically related conditions were named significantly faster than the unrelated condition (syllable-related: $t_{(18.4)}=8.23, p_{\text {adjusted }}<0.001$, cohen' $s d=-0.58$; initial segment-related: $t_{(16.8)}=$ $2.94, p_{\text {adjusted }}=0.014$, cohen's $d=-0.23$; final segment-related: $t_{(16.7)}=2.27, p_{\text {adjusted }}=0.037$, cohen's $\left.d=-0.21\right)$.

\section{Electrophysiological Results}

One participant was excluded from the EEG analysis because of large electrode drift and excessive artefacts. Besides, incorrect responses, naming latencies shorter than $500 \mathrm{~ms}$ or longer than $1500 \mathrm{~ms}$ was excluded in ERP data analysis.

Time windows were chosen based on results of an analysis of consecutive 10-ms time windows. First, the three phonologically related conditions at each ROI were compared with the unrelated condition via serial paired $t$-tests with a step size of $10 \mathrm{~ms}$. The time windows were chosen when at least five consecutive $t$-tests approached significance $(p<$ 0.05 , two tailed) for each related condition at one ROI.

Three time windows were selected ( $320 \mathrm{~ms}$ to $500 \mathrm{~ms}$ post pictures onset for the syllable-related condition, $370 \mathrm{~ms}$ to $420 \mathrm{~ms}$ for the initial segment-related condition, and $400 \mathrm{~ms}$ to $450 \mathrm{~ms}$ for the final segment-related condition). In each time window, an omnibus ANOVA was conducted included distractor type (syllable-related or initial segment-related or final segment-related vs. unrelated) and ROI. For the syllable-related and unrelated comparison in the time windows 
of 320-500 ms, the main effects of distractor type and ROI were significant (distractor type: $F(1,18)=4.78, p<0.05$; ROI: $F(8,144)=10.43, p<0.001)$, and the interaction between distractor type and ROI was also significant $(F(8,144)$ $=3.88, p<0.001)$. For the initial segment-related and unrelated comparison in the time window of $370-420 \mathrm{~ms}$, the main effects of distractor type and ROI were significant (distractor type: $F(1,18)=4.67, p<0.05$; ROI: $F(8,144)=$ $11.45, p<0.001)$, and the interaction between distractor type and ROI was not significant $(F(144,8)=1.71, p=0.1)$. For the final segment-related and unrelated comparison in the time window of 400-450 ms, the main effect of distractor type was not significant, while the main effect of ROI was significant (distractor type: $F(18,1)=2.3, p=0.146$; ROI: $F(144,8)=13.89, p<0.001)$, and the interaction between distractor type and ROI was only marginally significant $(F$ $(144,8)=1.87, p=0.069)$

Furthermore, each of the three phonologically related condition was compared to the unrelated condition in each ROI, and $f d r$ correction was applied to avoid the multiple comparison problem. For the syllable-related and unrelated comparison in the time window of 320-500 ms, four ROIs showed significant difference: middle anterior $(t(18)=2.71$, $p_{\text {adjusted }}=0.043$, cohen's $\left.d=0.62\right)$, right anterior $\left(t(18)=3.11, p_{\text {adjusted }}=0.042\right.$, cohen's $\left.\mathrm{d}=0.71\right)$, right middle $(t(18)$ $=2.91, p_{\text {adjusted }}=0.042$, cohen's $\left.d=0.67\right)$ and right posterior $\left(t(18)=2.51, p_{\text {adjusted }}=0.049\right.$, cohen 's $\left.d=0.57\right)$. For the initial segment-related and unrelated comparison in the time window of 370-420 ms, results showed significant (or marginally significant $)$ in the left anterior $\left(t(18)=2.15, p_{\text {adjusted }}=0.082\right.$, cohen's $\left.d=0.49\right)$, middle anterior $(t(18)=2.66$, $p_{\text {adjusted }}=0.048$, cohen's $\left.d=0.61\right)$, right anterior $\left(t(18)=2.39, p_{\text {adjusted }}=0.062\right.$, cohen' $\left.s d=0.55\right)$, middle central $(t(18)$ $=2.69, p_{\text {adjusted }}=0.048$, cohen's $d=0.62$ ). For the final segment-related and unrelated comparison in the time window of 400-450 ms, four ROIs showed significant (or marginally significant) difference: right middle $(t(18)=2.37$, $p_{\text {adjusted }}=0.066$, cohen's $\left.d=0.54\right)$, left posterior $\left(t(18)=2.81, p_{\text {adjusted }}=0.052\right.$, cohen's $\left.d=0.64\right)$, middle posterior $(t(18)$ $=2.42, p_{\text {adjusted }}=0.066$, cohen's $\left.d=0.56\right)$ and right posterior $\left(t(18)=3.67, p_{\text {adjusted }}=0.016\right.$, cohen 's $\left.d=0.84\right)$. Averaged ERP waveforms of three typical ROIs and detailed results of paired t test for each comparison in each ROI are displayed in Fig 1D and Fig 1E.

To identify time points at which the ERPs between two conditions (related versus unrelated) begin to diverge, onset latency analysis was adopted with the method developed by Guthrie and Buchwald (Guthrie, Buchwald, 1991). This method was designed to prevent erroneous detections of short significant intervals through computer simulation. An interval would be declared to be significant if its observed number of consecutive significant time points is larger than the estimated time interval, and the initial time point would be taken as the starting point of an effect. The GuthrieBuchwald method was adopted to perform the onset latency analysis for each phonologically related condition. According to results of mean amplitude analyses, the electrode Fz was selected as the representative electrode for syllable-related condition and the initial segment-related condition, while P4 was selected to represent the final segment-related condition. Table 1 shows results for onset latencies analysis in different phonologically related conditions versus unrelated condition, respectively. For the syllable-related condition, the starting point was $322 \mathrm{~ms}$ post pictures onset and the effect lasted for $178 \mathrm{~ms}$; for the initial segment-related condition, the starting point was 368 $\mathrm{ms}$ and the effect lasted for $96 \mathrm{~ms}$; for the final segment-related condition, the starting point was $408 \mathrm{~ms}$ and the effect lasted for $52 \mathrm{~ms}$.

\section{Discussion}

The current study investigated the time courses of syllabic and segmental encoding during an overt spoken production via a PWI paradigm. Naming latencies of all three phonologically related conditions were significantly shortened compared to unrelated condition, reflecting the involvement of syllable and sub-syllabic units (such as initial segments 
and final segments) in spoken production. More importantly, ERP data provided a finely depiction on the time courses of processing different units: a syllabic effect from $320 \mathrm{~ms}$ to $500 \mathrm{~ms}$ on the middle anterior, right anterior, right middle and right posterior, an initial segmental effect from $370 \mathrm{~ms}$ to $420 \mathrm{~ms}$ mainly on the anterior area, and a final segmental effect from $400 \mathrm{~ms}$ to $450 \mathrm{~ms}$ on the left posterior, middle posterior, right posterior and right middle area. Onset latency analysis showed that the initiation of effects started from $322 \mathrm{~ms}$ for syllable, $368 \mathrm{~ms}$ for initial segment, and $408 \mathrm{~ms}$ for final segments, indicating a 46-ms interval from syllables to initial segments retrieval, and a 40-ms interval from initial to final segments retrievals.

The time intervals in which we found for syllable priming $(320-500 \mathrm{~ms})$, the initial segment-related priming (370-420 $\mathrm{ms}$ ), and the final segment-related priming (400-450ms) are roughly in agreement with the time course of phonological encoding, i.e., 275-455 ms (Indefrey, Levelt, 2004; Indefrey, 2011). Mean latencies in our study were about 730 ms, which is somewhat slower than $600 \mathrm{~ms}$ in the meta-analysis estimated previously (Indefrey, Levelt, 2004; Indefrey, 2011). The temporal course of syllabic and segmental encoding is broadly in line with results of studies in alphabetic languages and Mandarin Chinese with ERP methodology. For example, Wang et al. found a syllable priming effect in the 200-400 ms interval in Chinese spoken production using a delayed naming task (Wang, Wong, Wang \& Chen, 2017), which they interpreted as implying syllables as proximate units of phonological encoding. With regard to location, syllable priming effect presented at right anterior and posterior areas, an initial segment priming effect presented at left and right anterior areas, while a final segment priming effect presented at left and right posterior areas, suggesting that distinct EEG patterns involved for syllabic, initial and final segmental encoding. Specifically, all phonologically related conditions elicit less negative waveforms in comparison with unrelated condition, which is consistent with previous findings about phonological effects(Wang, Wong, Wang \& Chen, 2017; Yu, Mo, \& Mo, 2014 ; Wong, Wang \& Chen, 2016; Zhu, Damian \& Zhang, 2015; Dell'acqua et al., 2010).

Critically, we firstly observed that the syllable effect was $46 \mathrm{~ms}$ earlier than the initial segments effect. According to the WEAVER++ model incorporated with the proximate unit principle, syllabic "chunks" are retrieved at an early stage of word-form encoding, and then the segments of the syllable are retrieved. The $46 \mathrm{~ms}$ interval indicated that syllables are retrieved $46 \mathrm{~ms}$ before segments retrieval in word-form encoding. There are two possibilities to explain this time interval. One is that it takes $46 \mathrm{~ms}$ to retrieve a syllable ( 3 phonemes) for Chinese speakers, which was in line with previous finding (Wheeldon and Levelt, 1995; Turennout, Hagoort, \& Brown, 1998). Wheeldon and Levelt found that in phoneme monitoring, the reaction time difference between the onset and the offset of the first CVC syllable (3 phonemes) was $55 \mathrm{~ms}$ (Wheeldon and Levelt, 1995). van Turennout et al. found a slightly larger $80 \mathrm{~ms}$ difference between monitoring a word's onset and its offset in a dual-choice go/nogo task (Turennout, Hagoort, \& Brown, 1998), but with targets which were 50\% longer than those of Wheeldon and Levelt. An alternative is that the retrieval of segments initiates while the retrieval of syllables is not completed, which may result in a cascaded pattern for syllabic and segmental encoding in producing a Chinese word. These findings suggest that atonal syllables are the primary functional units of phonological encoding below the words level in Chinese spoken word production, and thus provide strong evidence for the proximate units principle.

However, the onset latencies showed that initial segments were retrieved $40 \mathrm{~ms}$ before final segments, which was contradicted with the parallel processing proposition (O'Seaghdha, Chen, \& Chen, 2010; Roelofs, 2015). Model of spoken production assumes that a morpheme's segments or phonemes become simultaneously available, but with labeled links indicating their correct ordering (Turennout, Hagoort, \& Brown, 1998). Wang, Wong \& Chen investigated this question with the PWI paradigm, and found that the facilitation effect of shared atonal syllables was elicited when distractors were presented $100 \mathrm{~ms}$ earlier (SOA: $-100 \mathrm{~ms}$ ) or simultaneously (SOA: $0 \mathrm{~ms}$ ) with the target picture, while 
the facilitation effect of sharing word-initial segments only appeared at $0 \mathrm{~ms} \mathrm{SOA}$, and null effects were found for sharing word-final segments, suggesting a serial processing order on segments (Wang, Wong, \& Chen, 2018).

Furthermore, Indefrey \& Levelt proposed that it takes $20 \mathrm{~ms}$ per phoneme during syllabification process in alphabetic langauges (Indefrey, Levelt, 2004; Indefrey, 2011). A $40 \mathrm{~ms}$ time interval between initial and final segments is nicely in line with their estimation. We therefore suggest that Chinese speakers processed the content of segments and their orders after syllables retrieval, and the spelled-out segments are successively inserted into the present metrical template. The sequential of initial and final segments in the present study could reflect a combination of segments and their position at a later stage of word-form encoding.

It should be noted that this interpretation is not in line with findings in previous studies. Yu, Mo, and Mo found that both the initial and non-initial phonemes modulate ERPs in the 180- to 300-ms interval, and they suggest a parallel explanation (Yu, Mo, \& Mo, 2014). However, the time interval they selected begins from $180 \mathrm{ms,} \mathrm{which} \mathrm{is} \mathrm{too} \mathrm{early} \mathrm{for}$ word-form encoding stage in spoken word production. Meanwhile, the $p$ values in Yu et al.'s study were not corrected in multiple comparison. We examined their $p$ values and found that all $p$ values were not significant via $f d r$ correction method. This problem was avoided in the present study.

The present study found reliable syllable priming effect and sub-syllabic segments priming effect simultaneously on naming latencies using a typical picture-word interference paradigm (Wong, Chen, 2008; Wang, Wong, Chen, 2018; Wong, Chen, 2009). By contrast, previous studies using various tasks consistently found syllable shared effect but no phoneme overlap effect. For example, using a form preparation (or implicit priming paradigm), J.-Y. Chen et al. reported a syllable benefit but no first consonant preparation benefit (Chen, Chen \& Dell, 2003, see also Chen, Chen' 2013). Using a masked priming task, J.Y. Chen et al. reported a facilitation effect in the syllable sharing condition, and a weakly negative effect in the initial consonants sharing condition (Chen, O'seaghdha, \& Chen, 2016). According to these comparisons, we suggest that picture-word interference task could be more sensitive to sub-syllabic segments overlap than other paradigms. Another factor affecting the sub-syllabic segments effect on naming latencies is the number of overlap phonemes between primes and targets. Further replication is need to examine this hypothesis. To summarize, the current study firstly provides a fine temporal course of syllable and segments encoding in Chinese spoken naming. Syllables are retrieved $46 \mathrm{~ms}$ before segments, and segments and their order are retrieved incrementally from left to right in producing Chinese spoken words. Our findings provide evidence for that phonological units vary across language in spoken production.

Table 1. Results of onset latency analysis for each comparison

\begin{tabular}{|c|c|c|c|}
\hline Comparison & $\begin{array}{l}\text { Suggested minimum } \\
\text { consecutive time points }\end{array}$ & $\begin{array}{c}\text { Consecutively significant } \\
\text { time points }(\max )\end{array}$ & Interval onset Latency \\
\hline $\begin{array}{c}\text { Syllable-related } v s . \\
\text { Unrelated }\end{array}$ & 14 & 89 & 322 \\
\hline $\begin{array}{c}\text { Initial segment-related } \\
\qquad v s . \text { Unrelated }\end{array}$ & 16 & 48 & 368 \\
\hline $\begin{array}{c}\text { Final segment-related } v s . \\
\text { Unrelated }\end{array}$ & 12 & 26 & 408 \\
\hline
\end{tabular}


A

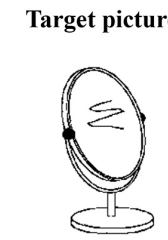

镜子 mirror /jing4 zi/

$\mathrm{D}$

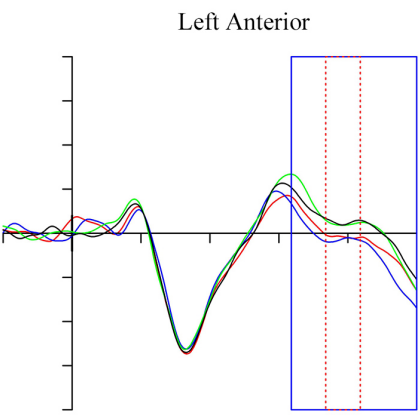

$\mathrm{B}$

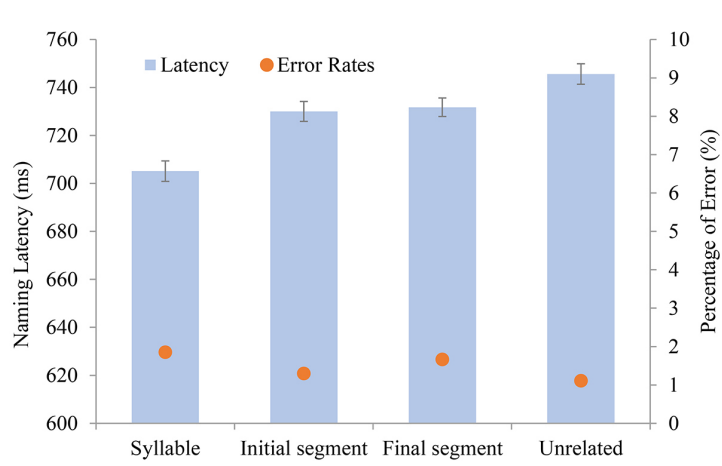

Syllable Initial segment Final segment Unrelated
$\mathrm{C}$

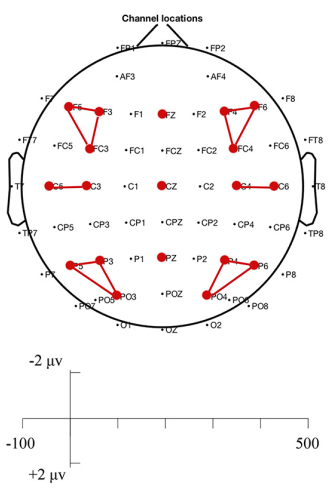

Right Anterior
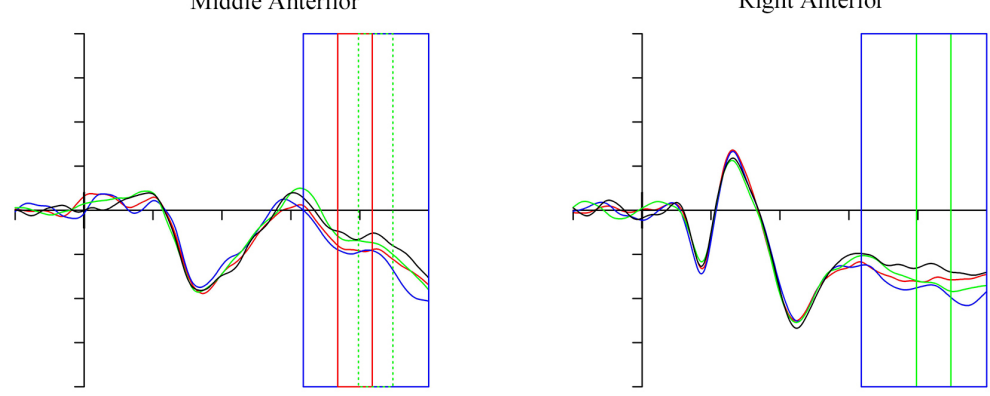

$\begin{array}{llll} & \text { Syllable-related } & & \text { Final Segment-related } \\ & \text { Initial Segment-related } & \text { Unrelated }\end{array}$

$\mathrm{E}$

Syllable-related $320-500 \mathrm{~ms}$

Initial-segment related $370-420 \mathrm{~ms}$

Final-segment related $400-450 \mathrm{~ms}$
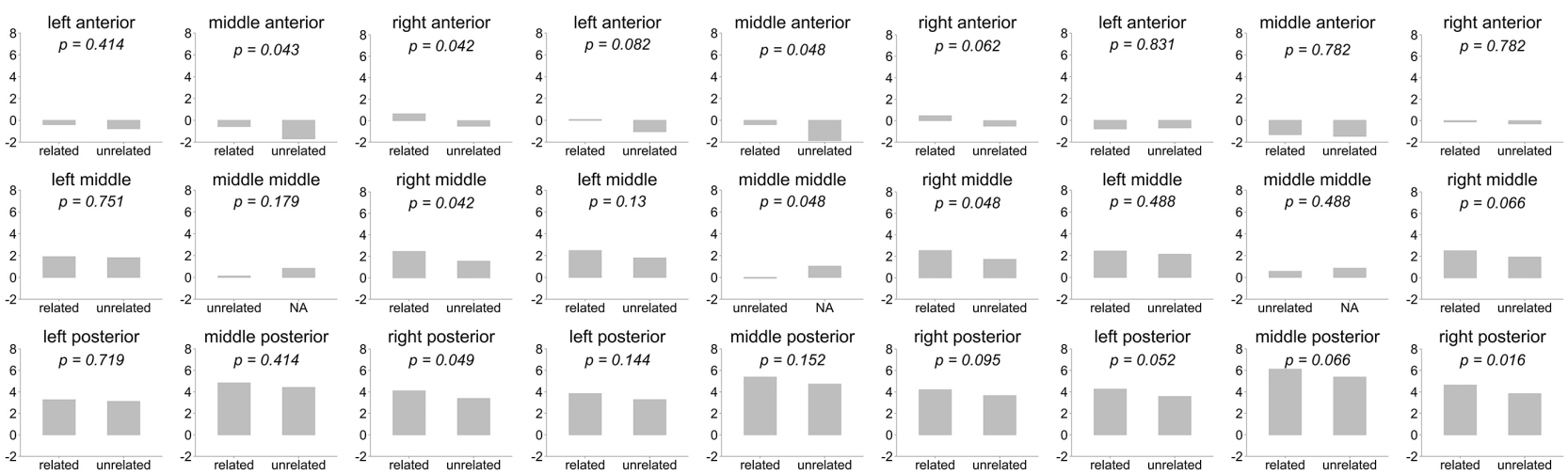

Figure 1. A) Instance of stimulus in this study with a target picture and its four corresponding distractors B) Average latencies in each condition. All three phonological related condition have shorter latencies than the unrelated condition. Error bars represent standard errors. C) Regions of interest and scale of axis D) Grand average ERPs for the four phonologically related/unrelated conditions (Blue: syllable-related; Red: initial segment-related; Green: final segmentrelated; Black: unrelated) at three ROIs: right-anterior ( $\mathrm{F} 4, \mathrm{~F} 6, \mathrm{FC} 4)$, right-middle $(\mathrm{C} 4, \mathrm{C} 6)$ and right-posterior $(\mathrm{P} 4, \mathrm{P} 6$, PO4). Rectangles with colors corresponding to conditions are used to mark significance (solid line) or marginal significance (dash line). E) Results of paired t-test for each comparison at corresponding time windows. All $p$ values are corrected with the $f d r$ method. 
Data availability. The data of the current study are available from the corresponding author upon reasonable request.

\section{Reference}

1. Levelt W.J.M, Roelofs A, Meyer AS (1999) A theory of lexical access in speech production. Behav Brain Sci 22:138, discussion 38-75.

2. Chen, J.-Y., Lin, W.-C., \& Ferrand, L. (2003). Masked priming of the syllable in Mandarin Chinese speech production. Chinese Journal of Psychology, 45, 107-120.

3. Oseaghdha, P. G., Chen, J. Y., \& Chen, T. M. (2010). Proximate Units in Word Production: Phonological Encoding Begins with Syllables in Mandarin Chinese but with Segments in English. Cognition, 115(2), 282-302.

4. Schiller, N. O. (1998). The effect of visually masked syllable primes on the naming latencies of words and pictures. Journal of Memory and Language, 39(3), 484-507.

5. Schiller, N. O. (1999). Masked syllable priming of English nouns. Brain and Language, 68(1), 300-305.

6. Schiller, N. O. (2000). Single word production in English: the role of subsyllabic units during phonological encoding. Journal of Experimental Psychology: Learning, Memory and Cognition, 26(2), 512-528.

7. Verdonschot, R. G., Kiyama, S., Tamaoka, K., Kinoshita, S., La Heij, W., \& Schiller, N. O. (2011). The Functional Unit of Japanese Word Naming: Evidence from Masked Priming. Journal of Experimental Psychology: Learning, Memory and Cognition, 37(6), 1458-1473.

8. Meyer, A. S. (1991). The time course of phonological encoding in language production: Phonological encoding inside a syllable. Journal of Memory and Language, 30, 69-89.

9. Meyer, A. S., \& Schriefers, H. (1991). Phonological facilitation in picture-word interference experiments: Effects of stimulus onset asynchrony and types of interfering stimuli. Journal of Experimental Psychology: Learning, Memory, and Cognition, 17, 1146-1160.

10. Roelofs, A. (1999). Phonological Segments and Features as Planning Units in Speech Production. Language and Cognitive Processes, 14(2), 173-200.

11. Schiller, N. O. (2004). The onset effect in word naming. Journal of Memory and Language, 50(4), 477-490.

12. Damian, M. F., \& Dumay, N. (2007). Time pressure and phonological advance planning in spoken production. Journal of Memory and Language, 57, 195-209.

13. Collins, A. F., \& Ellis, A. W. (1992). Phonological priming of lexical retrieval in speech production. British Journal of Psychology, 83(3), 375-388.

14. Damian, M. F., \& Dumay, N. (2009). Exploring phonological encoding through repeated segments. Language and Cognitive Processes, 24(5), 685-712.

15. Timmer, K., Ganushchak, L. Y., Mitlina, Y., \& Schiller, N. O. (2014). Trial by trial: selecting first or second language phonology of a visually masked word. Language, cognition and neuroscience, 29(9), 1059-1069.

16. Jouravlev, O., Lupker, S. J., \& Jared, D. (2014). Cross-language phonological activation: evidence from masked onset priming and ERPs. Brain and Language, 11-22.

17. Timmer, K., Vahidgharavi, N., \& Schiller, N. O. (2012). Reading aloud in Persian: ERP evidence for an early locus of the masked onset priming effect. Brain and Language, 122(1), 34-41

18. Chen, J. Y., Chen, T. M., \& Dell, G. S. (2002). Word-Form Encoding in Mandarin Chinese as Assessed by the Implicit Priming Task. Journal of Memory and Language, 46(4), 751-781. 
19.Zhang, Q. (2008). Phonological Encoding in Monosyllabic and Bisyllabic Mandarin Word Production: Implicit Priming Paradigm Study: Phonological Encoding in Monosyllabic and Bisyllabic Mandarin Word Production: Implicit Priming Paradigm Study. Acta Psychologica Sinica, 40(3), 253-262.

20. You, W., Zhang, Q., \& Verdonschot, R. G. (2012). Masked Syllable Priming Effects in Word and Picture Naming in Chinese. PLOS ONE, 7(10).

21. Wang, J., Wong, A. W., Wang, S., \& Chen, H. (2017). Primary phonological planning units in spoken word production are language-specific: Evidence from an ERP study. Scientific Reports, 7(1).

22. Chen, J. Y., Oseaghdha, P. G., \& Chen, T. M. (2016). The primacy of abstract syllables in Chinese word production. Journal of Experimental Psychology: Learning, Memory and Cognition, 42(5), 825-836.

23. Chen, T. M., \& Chen, J. Y. (2013). The syllable as the proximate unit in Mandarin Chinese word production: An intrinsic or accidental property of the production system? Psychonomic Bulletin \& Review, 20(1), 154-162.

24. Wong, A. W. K., \& Chen, H.-C. (2008). Processing segmental and prosodic information in Cantonese word production. Journal of Experimental Psychology: Learning, Memory, and Cognition, 34, 1172-1190.

25.Qu, Q., Damian, M. F., \& Kazanina, N. (2012). Sound-sized segments are significant for Mandarin speakers. Proceedings of the National Academy of Sciences of the United States of America, 109(35), 14265-14270.

26. Yu, M., Mo, C., \& Mo, L. (2014). The role of phoneme in mandarin Chinese production: Evidence from ERPs. PLOS ONE, 9(9), e106486.

27. Yu, M., Mo, C., Li, Y., \& Mo, L. (2015). Distinct representations of syllables and phonemes in Chinese production: Evidence from fMRI adaptation. Neuropsychologia,, 253-259.

28. Oseaghdha, P. G. (2015). Across the great divide: Proximate units at the lexical-phonological interface. Japanese Psychological Research, 57(1), 4-21.

29. Roelofs, A. (2015). Modeling of phonological encoding in spoken word production: From Germanic languages to Mandarin Chinese and Japanese. Japanese Psychological Research, 57(1), 22-37.

30. Laganaro, M., Valente, A., \& Perret, C. (2012). Time course of word production in fast and slow speakers: a high density ERP topographic study. NeuroImage, 59(4), 3881-3888.

31. Roelofs, A., \& Shitova, N. (2017). Importance of response time in assessing the cerebral dynamics of spoken word production: Comment on Munding et al. (2016). Language, cognition and neuroscience, 32(8), 1064-1067.

32. Wang, J., Wong, A. W., \& Chen, H. (2018). Time course of syllabic and sub-syllabic processing in Mandarin word production: Evidence from the picture-word interference paradigm. Psychonomic Bulletin \& Review, 25(3), 11471152.

33. YUE Yuan; ZHANG Qingfang. Syllable and Segments Effects in Mandarin Chinese Spoken Word Production. Acta Psychologica Sinica, 2015, 47(3): 319-328.

34. Bates, D. (2005). Fitting linear mixed models in R. R News, 5(1), Article 5. Retrieved from http://ftp.cs.pu.edu.tw/network/CRAN/doc/Rnews/Rnews_2005-1.pdf.

35. Baayen, R.H., Davidson, D.J., \& Bates, D.M. (2008). Mixed-effects modeling with crossed 576 random effects for subjects and items. J. Mem. Lang. 59, 390-412. doi: 577 10.1016/j.jml.2007.12.005

36. Barr, D.J., Levy, R., Scheepers, C., \& Tily, H.J. (2013). Random effects structure for confirmatory hypothesis testing: Keep it maximal. J. Mem. Lang. 68, 255-278. doi: http://dx.doi.org/10.1016/j.jml.2012.11.001

37. R Development Core Team (2009). R: A language and environment for statistical computing. (version 2.8.1). [Software]. (Vienna, Austria: R Foundation for Statistical Computing, http://www.R-project.org).

38. Guthrie, D., \& Buchwald, J. S. (1991). Significance testing of difference potentials. Psychophysiology, 28, $240-244$. 
39. Indefrey, P., \& Levelt, W. J. M. (2004). The spatial and temporal signatures of word production components. Cognition, 92, 101-144.

40. Indefrey, P. (2011). The spatial and temporal signatures of word production components: A critical update. Frontiers in Psychology, 2, 1-16. http://dx.doi.org/ 10.3389/fpsyg.2011.00255.

41. Wong, A. W., Wang, J., Ng, T., \& Chen, H. (2016). Syllabic encoding during overt speech production in Cantonese: Evidence from temporal brain responses. Brain Research, 101-109.

42.Zhu, X., Damian, F., \& Zhang, Q*. (2015). Seriality of semantic and phonological processes during overt speech in Mandarin as revealed by event-related brain potentials. Brain and Language, 144, 16-25

43. Dellacqua, R., Sessa, P., Peressotti, F., Mulatti, C., Navarrete, E., \& Grainger, J. (2010). ERP evidence for ultra-fast semantic processing in the picture-word interference paradigm. Frontiers in Psychology, 177-177.

44. Wheeldon, L., \& Levelt, W. J. (1995). Monitoring the time course of phonological encoding. Journal of Memory and Language, 34(3), 311-334.

45. Van Turennout, M., Hagoort, P., \& Brown, C. M. (1998). Brain Activity During Speaking: From Syntax to Phonology in 40 Milliseconds. Science, 280(5363), 572-574.

46. Levelt, W. J. M. (1999). Models of word production. Trends in Cognitive Sciences, 3(6), $223-232$.

47. Wong, A. W., \& Chen, H. (2009). What are effective phonological units in Cantonese spoken word planning. Psychonomic Bulletin \& Review, 16(5), 888-892.

48. Chen, T. M., \& Chen, J. Y. (2013). The syllable as the proximate unit in Mandarin Chinese word production: An intrinsic or accidental property of the production system? Psychonomic Bulletin \& Review, 20(1), 154-162.

49.Zhang, Q., \& Yang, Y. (2003). The determiners of picture-naming latency (in Chinese). Acta Psychologica Sinica, 35(4), 447-454. 


\section{Acknowledgements}

This work was supported by the National Natural Science Foundation of China (31471074), the Key Project of Beijing Social Science Foundation in China (16YYA006), the Fundamental Research Funds for the Central Universities, and the Research Funds of Renmin University of China (18XNLG28) to Qingfang Zhang.

\section{Author Contributions}

Qingfang Zhang designed the experiment. Yuan Yue conducted the experiment. Chen Feng analyzed the data. Chen Feng and Qingfang Zhang wrote the manuscript.

Competing Interests: The authors declare that they have no competing interests. 